MOGLICHKEITEN UND GRENZEN EINER STAATLICHEN DEZENTRALISIERUNGSPOLITIK IM BEREICH DER LANDESVERTEIDIGUNG DER BUNDESREPUBLIK DEUTSCHLAND

Herman Baer*

IZVLEČK

UDK $911.37: 711.2: 725.181(430.1)=30$

MOŽNOSTI IN MEJE DRŽAVNE POLITIKE DECENTRALIZACIJE NA PODROČJU NARODNE OBRAMBE

Clanek govori o pozitivnih predvsem gospodarskih in o negativnih predvsem prebivalstvenih ter drugih posledicah obrambne funkcije $v$ obrobnih območjih ter vpliv tega na razvoj manj razvitih območij.

ABSTRACT

UDC $911.37: 711.2: 725.181(430.1)=30$

THE POSSIBILITIES OF STATE POLICY DECENTRALISATION ON THE FIELD OF NATIONAL DEFENCE

The item deals with positive - above all economic and negative - above all populational and other consequences of defence function in peripheral zones and their influence on less developed zones.

Eingebunden in das Konzept einer regionalen Regionalpolitik ist der Teilaspekt der Dezentralisierung staatlicher Einrichtungen. Innerhalb dieser Massnahmen habe ich mich mit den Möglichkeiten und den Grenzen im Bereich der Landesverteidigung beschäftigt, die ich Ihnen skizzieren möchte.

Zur Problematik der Dezentralisierung militärischer Einrichtungen

Sie werden mit Recht skeptisch sein und sich fragen, ob es denn realistisch sei, gerade diesen staatlichen Bereich in derartige Dezentralisierungsubberlegungen einzubeziehen, geht es doch im Falle der Landesverteidigung - und dies gilt wohl auch für Ihr Land - in erster Linie um die Gewährleistung der nationalen Sicherheit, in unserem Land darüberhinaus um die Erfüllung supranationaler, bündnispolitischer Inte-

* Dipl.geogr., Lehrstuhl für Wirtschaftsgeographie und Raumplanung, Universităt Bayreuth, Universitătsstrasse 31 
ressen, und nicht etwa um die Belange von Teilregionen des Gesamtstaates, so berechtigt deren Anliegen im einzelnen auch sein mögen.

Stehen sich also hier im Grunde nicht - ich will nicht sagen zwei völlig konträre - aber doch unterschiedlich gelagerte Interessensfelder mit ganz spezifischen Aufgaben und Zielen gegenüber: Auf der einen Seite die Gewährleistung der Sicherheit des Gesamtstaates, die erst die Voraussetzungen fur alle anderen Bemuhungen innerhalb eines Staates, $z$. B. auch um den Ausgleich regionaler Disparităten schafft - und auf der anderen Seite das spezifische Interesse einer Region, das den Gesamtstaat zwar nicht negiert, aber mit seiner kleinräumigen und auf die Entwicklung endogener Potentiale abzielenden Denkweise die regionale Komponente doch bewusst und nachhaltig betont.

Hinzu kommt, dass wir, wenn wir von Dezentralisierung sprechen, nicht nur eine blosse Dekonzentration von Einrichtungen und Mitteln meinen, etwa in der Art vieler Zweigbetriebe, die ohne eigene Entscheidungsbefugnisse ausgestattet, eine hohe Konjunkturempfindlichkeit aufweisen und für eine Region deshalb kaum einen dauerhaften Entwicklungsimpuls darstellen, sondern die Abhängigkeit von den Entscheidungszentren, die vielfach mit den Verdichtungsrăumen identisch sind, eher noch erhöhen.

Wir gehen dagegen bei dem Ansatz einer regionalen Regionalpolitik davon aus, dass die Bemühungen um eine Stärkung strukturschwacher Regionen nur dann erfolgreich sein können, wenn damit gleichzeitig auch eine Dezentralisierung von Entscheidungsstrukturen verbunden ist, da eine regionale Regionalpolitik nur dann durchsetzbar ist, wenn für eine Region bedeutsame Entscheidungen auch in der Region selbst gefällt werden können.

Bei unserem Vèrstăndnis von Dezentralisierung ergeben sich somit, angewandt auf den Bereich der Landesverteidigung Probleme in zweierlei Hinsicht:

zum einen aufgrund des spezifischen Charakters dieser staatlichen Aufgabe und zum anderen aber auch aus regionalpolitischer Sicht, bedeutet doch Dezentralisierung für die profitierende Region ein Mehr an Entscheidungsbefugnis und damit letztlich auch an Macht und für andere Regionen dagegen möglicherweise den Verlust an Gewicht und Einfluss.

Nach diesen allgemeinen Ausfuhrungen zur Problemstellung lassen Sie mich nun zu den raumwirksamen Aspekten der Landesverteidigung kommen, um sodann auf den Zusammenhang zwischen Landesverteidigung und Raumplanung einzugehen und dabei Möglichkeiten und Grenzen des Einsatzes der Landesverteidigung als Instrument der Raumplanung zu diskutieren. 


\section{Raumwirksame Aspekte der Landesverteidigung}

Als Raumwirksamkeit der Landesverteidigung sollen hier all diejenigen Auswirkungen verstanden werden, die entweder direkt durch eine militărische Flächenbeanspruchung gegeben sind - denken Sie hier etwa nur an Kasernengelănde, Ubungsflächen, Flugplätze usw. - oder indirekt in Form regionalökonomischer Effekte, bedingt durch Ausgaben im Zusammenhang mit der Landesverteidigung in einer Region auftreten. Nicht zu vergessen sind dabei auch die Auswirkungen auf den regionalen Arbeitsmarkt etwa durch die Vergabe von Auftrăgen an die Wirtschaft oder aber auch durch das direkte Auftreten der Bundeswehr als ziviler Arbeitgeber.

Im Gegensatz zu dem Flăchenanspruch des Militărs, der nahezu von allen betroffenen Regionen als nachteilig empfunden wird, werden die regionalökonomischen und strukturpolitischen Effekte die von der Landesverteidigung ausgehen, meist als Positivum herausgestellt, erhofft man sich doch von der Dislozierung m litărischer Standorte positive Auswirkungen auf die regionale Wirtschaft und eine Verbesserung der regionalen und örtlichen Infrastrukturausstattung.

Um dies zu konkretisieren, möchte ich Ihnen am Beispiel des Garnisonsstandortes Bayreuth aufzeigen, welche Auswirkungen damit im einzelnen verbunden sind.

Beginnen möchte ich dabei mit den Auswirkungen auf den regionalen Arbeitsmarkt. Hier ergab unsere Untersuchung, dass im Bereich des Garnisonsstandortes Bayreuth allein rd. 580 Zivilbedienstete beschaftigt sind und das Milităr damit einer der bedeutendsten Arbeitgeber in der Stadt uberhaupt ist. Wichtig ist dabei auch, dass nahezu die Hälfte dieser zivilen Arbeitsplätze mit Frauen besetzt ist, hat es doch gerade diese $\mathrm{Be}$ völkerungsgruppe in lăndlichen Gebieten besonders schwer, einen Arbeitsplatz $\mathrm{zu}$ finden.

Bezüglich der regionalökonomischen Wirksamkeit der Personalausgaben, die mit rd. 64 Mio. DM im Jahr 1980 den jeweils grössten Anteil an den Gesamtetats der Garnisonen ausmachten, kann davon ausgegangen werden, dass rd. ein Drittel, dies entsprich etwa 28 Mio. DM dieser Ausgaben, im Nahbereich der Garnisonen verblieben. Von den dabei mittels einer Umfrage unter den Garnisonsangehorigen erfassten privaten Konsumausgaben in Höhe von rd. 27 Mio. DM entfielen ca. $60 \%$ auf den Einzelhandel, rd. $30 \%$ auf das örtliche Gaststättengewerbe und cca. $10 \%$ auf örtliche Tankstellen und das Kfz-Handwerk. Im Gaststättengewerbe waren es dabei insbesondere die in Bayreuth stationierten US-Soldaten, die einen entscheidenden Anteil ausmachten. 
Als ein weiterer regionalökonomisch wichtiger Bereich sind schliesslich die dezentralen Beschaffungen anzusehen. So wurden allein für die Bundeswehr-Garnison in Bayreuth bei einem Ausgabevolumen von rd. 6,5 Mio. DM im Jahr 1979 für Verpflegung, Bauunterhalt, Bewirtschaftung eine $80 \%$ ige Beteiligung der örtlichen und regionalen Wirtschaft ermittelt.

Als Gründe für das relativ günstige Abschneiden der ansässigen Wirtschaft sind sowohl im Falle der Konsumausgaben wie auch bei den dezentralen Beschaffungen die Zentralität Bayreuths zu nennen.

Bayreuth verfügt offensichtlich über eine hinreichende wirtschaftliche Kapazităt, um die im Zusammenhang mit den Garnisonen auftretenden Bedarfe zu decken. Auch wirkt șich hier offensichtlich das Fehlen eines höherrangigen Zentrums, etwa in der Grössenordnung Münchens, positiv auf die Beteiligung der ansåssigen Wirtschaft aus.

Bei allen wirtschaftlichen Vorteilen fur Bayreuth muss jedoch auch der erhebliche Flächenbedarf der Garnisonen in Rechnung gestellt werden, sind doch mit insgesamt $r d$. 560 ha über $8 \%$ des gesamten Stadtgebietes unter militärischer Nutzung. Dies entspricht etwa der vierfachen Fläche aller Park- und Grünlächen der Stadt.

$\mathrm{Da}$ es für die Beurteilung der Auswirkungen einer Garnison auf eine Stadt oder eine Region letztlich nicht genügt, allein die ökonomischen Effekte und die Flächenansprüche zu ermitteln, wurden zusätzlich Fragen der sozialen Integration von Garnisonsangehörigen in die Gemeinde untersucht. Wie die Untersuchung ergab, besteht dazu kein einheitliches Bild. Es zeigte sich nämlich, dass Wehrpflichtige und in den Kasernen untergebrachte Soldaten kaum in die Gemeinde integriert sind, wăhrend Soldaten, die ausserhalb der Kasernen wohnen, weitgehend in die Gemeinschaft der Standortgemeinde eingebunden sind.

Was die US-Soldaten betrifft, so kommt hier zu der Isolation der Kaserne noch die fremde Sprache und auch die nur kurze Verweildauer am Standort hinzu, die ejne Einbindung in die Stadt erschweren.

Geht man nach den vorliegenden Ergebnissen davon aus, dass Einrichtungen und Mittel der Landesverteidigung gegebenenfalls unter bestimmten Voraussetzungen dazu beitragen können, auch landesplanerische Ziele zu verfolgen, so stellt sich die Frage, inwieweit von seiten der Landesplanung Einflussmöglichkeiten oder gar ein Mitspracherecht bestehen, wenn es etwa um die Lozierung militärischer Einrichtungen geht. Damit aber ist unmittelbar das Verhältnis zwischen Raumplanung und militärischer Fachplanung angesprochen. 


\section{Zum Verhăltnis Raumplanung und militårische Fachplanung}

Die rechtlichen Grundlagen, die das Verhältnis von Raumplanung und militärischer Fachplanung bestimmen, sind durch das Bundesraumordnungsgesetz und dessen Konkretisierung in den Landesentwicklungsprogrammen und - plănen der Lănder auf der einen Seite - und den milită rischen Fachplanungsgesetzen auf der anderen Seite gegeben.

So ist zum Bereich der Verteidigung im Landesentwicklungsprogramm Bayern beispielsweise $u$. a. ausgesagt, dass die Belange der Verteidigung und der sonstigen Belande der Raumordnung sorgfältig gegeneinander und untereinander abzuwägen sind. Weiterhin heisst es auch, dass Truppenunterklinfte möglichst nur in geeigneten Zentralen Orten, in Gebieten errichtet werden sollen, deren Struktur zur Verbesserung der Lebensbedingungen gestärkt werden soll.

Von den militărischen Fachplanungsgesetzen sind es insbesondere das Landbeschaffungsgesetz und das Schutzbereichsgesetz, die für die Raumordnung bedeutsam sind, regeln diese Gesetze doch alle Massnahmen im Zusammenhang mit der Deckung des Landbedarfs und der Einschrănkung von Grundstücksnutzungen bei Vorhaben der Landesverteidigung.

Als Gesetze, die raumbedeutsam sind, enthalten sie sog. Raumordnungsklauseln, d. h. die Vorgabe, dass bei ihrer Anwendung ein Anhörungsverfahren durchzuführen ist, bei dem die Trăger offentlicher Belange hierzu Stellung nehmen können. Damit ist es für die Landesplanung möglich, uber die Raumordnungsklauseln in den entsprechenden Fachplanungsgesetzen an der militärischen Fachplanung formell beteiligt zu werden.

Es muss jedoch darauf hingewiesen werden, dass rechtlich gesehen militärische Einrichtungen mit sicherheitspolitischen Begründungen auch gegen den Widerstand der Raumplanung errichtet werden können. Dies bedeutet aber gleichzeitig, dass dem Einsatz der Landesverteidigung als Instrument der Landesplanung dort Grenzen gesetzt sind, wo es um das Primat sicherheitspolitischer Interessen geht. Damit ist die hăufig gestellte Forderung, Verteidigungsvorhaben als Instrument der Landesplanung einzusetzen, im Sinne einer gezielten Raumordnungspolitik, sicherlich überspitzt und wohl auch unrealistisch. Dies ändert jedoch nichts daran, dass Massnahmen und Einrichtungen im Zusammenhang mit der Landesverteidigung aus landesplanerischer Sicht optimal loziert werden sollten, soweit dies fachplanerische Uberlegungen zulassen. Hinzu kommt, dass in unserem Land zwar de jure die Möglichkeit besteht, militărische Vorhaben mit sicherheitspolitischen Argumenten durchzusetzen, die Realităt jedoch durchwegs so aussieht, dass die Verfügbarkeit von Grund 
und Boden heute, etwa bei der Lozierung militärischer Standorte, die entscheidende Rolle spielt und militärische Gesichtspunkte vielfach zurücktreten müssen.

Meine Damen und Herren, lassen Sie mich zum Abschluss noch eine Bemerkung anfügen. Sollte bei Ihnen durch meinen Vortrag der Eindruck entstanden sein, dass hier auf dem Umweg über eine Stärkung der regionalen Wirtschaft und des regionalen Arbeitsmarktes eine Forsierung militärischer Einrichtungen angestrebt wird, so möchte ich diesen Eindruck gerne korrigieren. Meine Uberzeugung ist, dass es mit Sicherheit nützlichere Bereiche gäbe, die enormen Mittel, die jăhrlich für Verteidigung ausgegeben werden, anzulegen. Ich bin jedoch auch der Meinung, dass man unter den gegebenen Voraussetzungen bemüht sein sollte, die Mittel der Verteidigung so einzusetzen, dass damit auch für andere Lebensbereiche positive Effekte erzielt werden können. 
korrigieren. Meine Uberzeugung ist, dass es mit Sicherheit nutzlichere Bereiche gäbe, die enormen Mittel, die jährlich für Verteidigung ausgegeben werden, anzulegen. Ich bin jedoch auch der Meinung, dass man unter den gegebenen Voraussetzungen bemuht sein sollte, die Mittel der Verteidigung so einzusetzen, dass damit auch für andere Lebensbereiche positive Effekte erzielt werden können.

Abb. 1 Regionalökonomische Wirksamkeit der Personalausgaben von Bundeswehr, Bundesgrenzschutz und US-Army in Bayreuth

Stand: $1979 / 80$

regionalökonomisch wirksamer Teil

der Personalausgaben

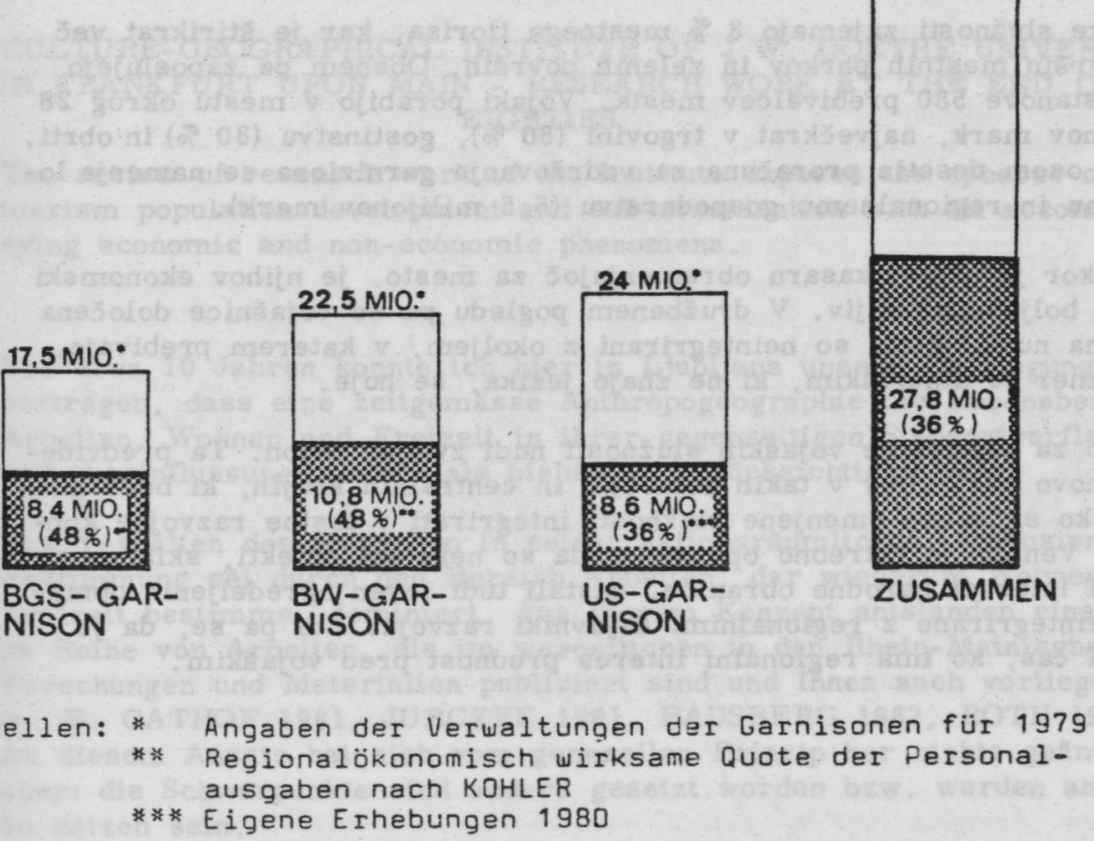




\section{MOŽNOSTI IN MEJE DRŽAVNE POLITIKE DECENTRALIZACIJE NA PODROČJU NARODNE OBRAMBE}

Nerealno bi bilo pričakovati, da bi se obrambna politika in njeno načrtovanje prilagodili potrebam in zahtevam posameznih regionalnih enot $\mathrm{v}$ ZR Nemčiji. Vendar bi smeli pričakovati, da bo načrtovanje novih vojaških objektov slonelo na razvojnih konceptih regije in da bo upoštevalo razpoložljive resurse. V tem smislu bi se naj izvršila decentralizacija vojaškega načrtovanja.

UČinek vojaških služnosti na prostor je dvojne narave: neposredno zajema obširne prostore $\mathrm{za}$ vojašnice, letališča, vojaške cone .... posredno pa krepi lokalno gospodarstvo. Gre za neposredna naročila (in plačila proizvajalcem opreme, hrane, storitvenim dejavnostim ipd.) ali za zagotavljanje delovnih mest in potrošnjo vojaškega osebja. Primer Bayreutha je dovolj ilustrativen.

Vojaške služnosti zajemajo $8 \%$ mestnega tlorisa, kar je štirikrat več od površin mestnih parkov in zelenih površin. Obenem pa zaposlujejo iste ustanove 580 prebivalcev mesta. Vojaki porabijo $\mathrm{v}$ mestu okrog 28 milijonov mark, največkrat v trgovini $(60 \%)$, gostinstvu $(30 \%)$ in obrti. Okrog osem desetin proračuna za vzdrževanje garniziona se namenja lokalnemu in regionalnemu gospodarstvu $(6,5$ milijonov mark).

V kolikor je obstoj kasarn obremenjujoč za mesto, je njihov ekonomski učinek bolj sprejemljiv. V družbenem pogledu pa so vojašnice določena socialna nuja: vojaki so neintegrirani z okoljem, v katerem prebivajo, pri čemer je ameriškim, ki ne znajo jezika, še huje.

Osnovo za planiranje vojaških služnosti nudi zvezni zakon. Ta predvideva njihovo postavitev $\mathrm{v}$ takih območjih in centralnih krajih, ki bodo gospodarsko sposobne omenjene služnosti integrirati $\mathrm{v}$ lastne razvojne koncepte. Vendar je potrebno opozoriti, da so nekateri objekti, skladno z višjimi interesi narodne obrambe, nastali tudi izven opredeljenih obmo$\chi_{i j}$, neintegrirano $\mathrm{z}$ regionalnimi dejavniki razvoja. Z di pa se, da je napočil čas, ko ima regionalni interes prednost pred vojaškim. 